\section{HEMORRHAGE AFTER TONSILLECTOMY.*} OSCAR WILKINSON, A.M., M.D.

WASHINGTON, $\mathfrak{D}$. C.

The infrequency of hemorrhage after tonsillectomy justifies me in reporting two recent cases.

CASE 1.-Mrs. S. came to me suffering with a follicular tonsillitis, with a history of frequent attacks of a similar kind. On examination I found the right tonsil very much more affected than the left, lying embedded between the tonsillar folds, to which it was slightly attached. It had a number of follicular cavities which were filled with a cheesy, offensive debris. As soon as the irritation had been relieved $I$ advised her to have the tonsil removed as a preventive of future attacks. The tonsil being small, it was easily raised from its bed with a pair of forceps. I seized it with a pair of forcepz, running through the ring of Mathew's tonsillotome, drew it through the opening and thus excised it. The tonsil was all removed and there was a slight peeling of the tonsillar pillars. The hemorrhage at first was not excessive, but it failed to stop as readily as is usual in such cases. I made a local ap. plication of adrenalin, 1 to 1,000 , which afforded some relief.

Having an engagement in Virginia at that hour, I was compelled to leave the patient with an assistant, with instructions to spray the tonsillar cavity with peroxid of hydrogen if the hemorrhage continued, and if this failed, to use compresses saturated in tannic acid and glycerin. I returned from Virginia in about an hour and found the patient almost completely. exsanguinated. The doctor had used adrenalin locally without effect, had sprayed the tonsillar cavity with hydrogen peroxid and had used compresses saturated in tannic acid and glycerin. This latter remedy had given temporary relief, forming a clot which, however, would soon be. pushed off by the hemorrhage from beneath. On examining the cavity carefully I saw that the hemorrhage oozed from the entire cut surface. I applied stiptic collodion without effect. I then resorted to Monsell's solution, which gave temporary relief, but within half an hour the clot became loose and the patient vomited abouit a pint of blood, and was very weak. Realizing that some more radical method must be attempted, I threaded a half curved eve needle with a black silk thread, and passing this needle through the posterinr tonsillar pillar and out through the anterior tonsillar pillar, was able, with the assistance of Dr. Stevenson, to draw this needle through. I then packed the tonsillar cavity behind this thread with a piece of cotton saturated in tannic acid and glycerin. I drew this thread tight, drawing the tonsillar pillars entirely together. The hemorrhage ceased and the patient had an uneventful recovery.

CASE 2.-Two weeks after my experience with the previous case $\overline{\mathrm{I}}$ operated on Mrs. M., removing her left tonsil, which was a rather small one of the follicular variety. In this case the hemorrhage stopped within a reasonable length of time. The patient walked home, a distance of six blocks, and as she entered the house her throat began to bleed. She attempted to check it by the use of ice gargles and hot solutions, but without success. I was called after she had been bleeding from two to two and one-half hours, and found her almost bloodless, being very weák and faint. I administered stimulants, gave adrenalin internally and applied it locally without avail. I used styptic collodion without results. I ordered a carriage and had her brought to my office. Immediately before leaving the house she vomited a considerable quantity of blood and was practically carried to the carriage. By the time she reached my office the hemorrhage had ceased and I had no further trouble with this case.

While I am an ardent advocate of the entire removal of diseased tonsillar tissue, my experience leads me to believe that we are more apt to have hemorrhage with our more radical operative procedures. The good results obtained justify complete removal, and with better instruments we are able to cope with the excessive hemorrhage when it does occur. I am aware that a different

\footnotetext{
*Read before the Fairfax County (Va.) Medical Society, June.
}

opinion has been expressed by good men, but these two cases of severe hemorrhage, and a similar one which I reported in the Baltimore Eye, Ear and Throat Journal in 1905, have all occurred in patients in whom a thorough tonsillectomy had been done. I have had made by the McKee Instrument Company a long-handled halfcurved needle with the eye in its point for use in such cases as described above.

\section{UTERUS FORCEPS. \\ GEORGE B. SOMERS, M.D. SAN FRANCISCO.}

In operating on the female pelvic organs through an abdominal incision, it is often necessary to grasp the uterus for the purpose of fixing, holding or elevating it.

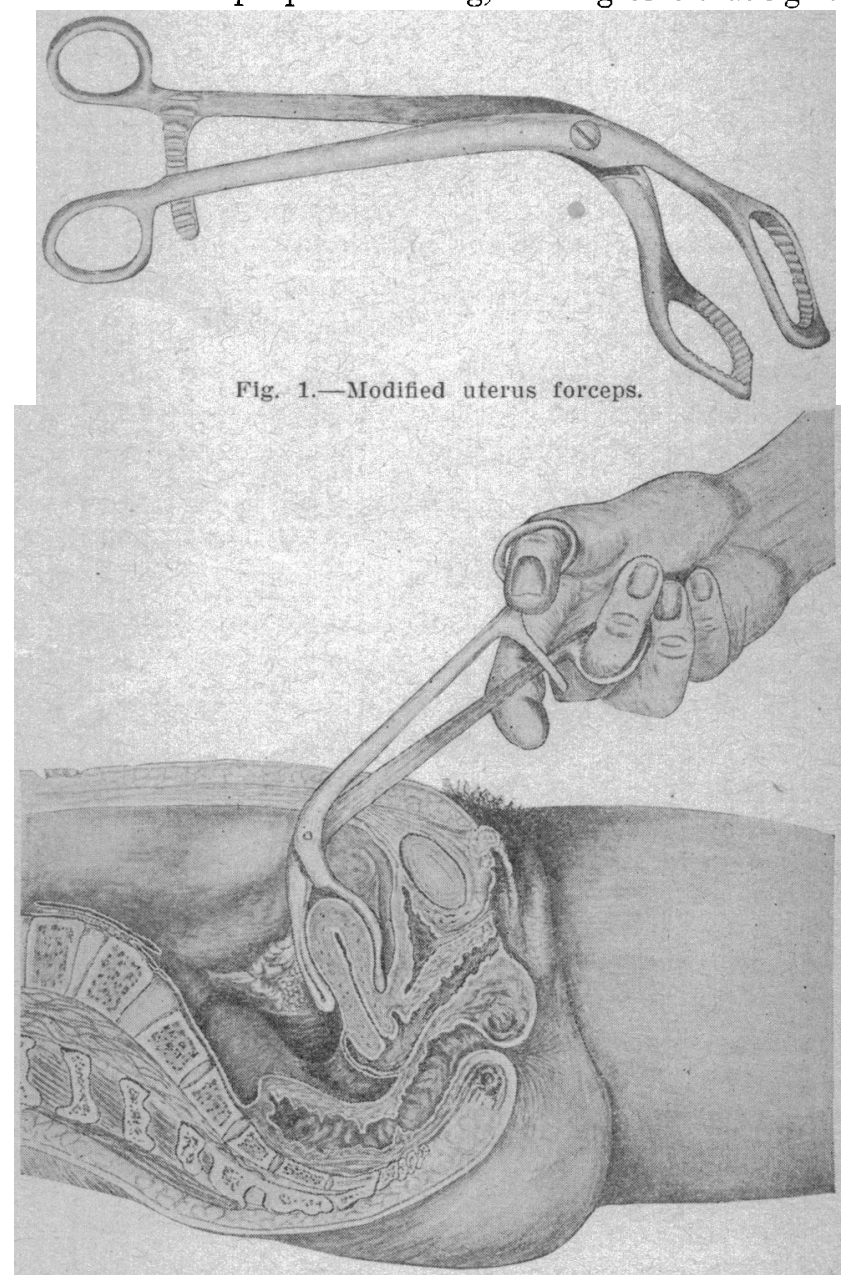

Fig. 2.-Uterus forceps applled.

The instruments commonly used are vulsella, which often lacerate the fundus so severely as to produce troublesome hemorrhage and cause a waste of time in repairing the injuries.

The only instrument that I know of, devised to hold the fundus without injuring it, is one described several years ago by Cullen of Baltimore. I found his instrument inadequate, as it does not grasp firmly enough and allows the uterus to slip. To hold the fundus firmly and with the least possible injury, I designed the instrument whose appearance. and use are sufficiently shown by the accompanying cuts.

It is needless to say that its chief use is in conservative work. Where a hysterectomy is to be performed, the injuries produced by vulsella may be disregarded. 